\title{
Acesso à Atenção Primária à Saúde de longevos: perspectiva de profissionais da Saúde da Família de um município do Rio Grande do Sul
}

\author{
Access to primary health care of elderly people: perspectives of \\ family health professionals in a municipality of Rio Grande do Sul
}

\author{
Cíntia Cristina Sulzbach', Teresinha Heck Weiller², Loiva Beatriz Dallepiane ${ }^{3}$ (D) \\ 'Programa de Pós-graduação em Gerontologia, Universidade Federal de Santa Maria (UFSM) - Palmeira das Missões (RS), Brasil. \\ 2Programa de Pós-graduação em Enfermagem, Universidade Federal de Santa Maria (UFSM) - Santa Maria (RS), Brasil. \\ ${ }^{3}$ Departamento de Alimentos e Nutrição, Universidade Federal de Santa Maria (UFSM) - Palmeira das Missões (RS), Brasil.
}

\begin{abstract}
Como citar: Sulzbach CC, Weiller TH, Dallepiane LB. Acesso à Atenção Primária à Saúde de longevos: perspectiva de profissionais da Saúde da Família de um município do Rio Grande do Sul. Cad Saúde Colet, 2020;28(3):373-380. https://doi.org/10.1590/1414-462X202028030158
\end{abstract}

\begin{abstract}
Resumo
Introdução: A Atenção Primária à Saúde é imprescindível no cuidado ao idoso longevo, devido à heterogeneidade e a especificidade dessa população, principalmente em relação ao acesso. Objetivo: Analisar a percepção sobre o acesso de longevos à Atenção Primária à Saúde na perspectiva dos profissionais da Estratégia Saúde da Família, em um município do Sul do Brasil. Método: Abordagem qualitativa, exploratório, do qual participaram dez profissionais vinculados à Estratégia Saúde da Família. Os dados foram coletados por meio de entrevista, cujas perguntas sobre acesso foram adaptadas e norteadas pelo Primary Care Assessment Tool, realizando-se análise temática de conteúdo. Resultados: Os participantes mencionaram como facilitadores do acesso a realização de visitas domiciliares, o acolhimento, o atendimento rápido e por demanda espontânea. Por outro lado, foram percebidas barreiras, como as unidades não estarem abertas em todos os momentos de necessidade dos longevos, falta de recepcionista, distância de outros serviços quando as unidades estão fechadas e falta de prioridade ao atendimento dos longevos. Conclusão: Concluímos que na percepção dos profissionais de saúde muitos fatores do acesso estão relacionados ao processo de trabalho e há uma compreensão limitada de que o atendimento ao longevo deve ser específico devido às alterações fisiológicas e sociais nessa população.
\end{abstract}

Palavras-chave: idoso; envelhecimento; acesso aos serviços de saúde; Sistema Único de Saúde; nutrição do idoso.

\begin{abstract}
Background: Primary health care is vital in providing attention to the elderly lifelong, due to the heterogeneity and the specificity of this population, particularly in relation to access. Objective: The objective of the present work was to analyze the perception of the oldest access to Primary Health Care from the perspective of professionals of the Family Health Strategy in a municipality in Southern Brazil. Method: A qualitative study and exploratory, attended by ten professionals linked to the Family Health Strategy was carried out. The data were collected by means of interviews, whose questions about access were based and adapted from Primary Care Assessment Tool and analyzed according to the precepts of Thematic Content Analysis. Results: The participants mentioned as facilitators of access to conducting visits, the reception and fast service and by spontaneous demand. On the other hand, there were perceived barriers, such as the units are not open at all times, lack of receptionist, far from
\end{abstract}

Trabalho realizado em Unidades de Saúde da Família - Palmeira das Missões (RS), Brasil.

Correspondência: Loiva Beatriz Dallepiane. E-mail: loiva.dallepiane@hotmail.com

Conflito de interesses: nada a declarar.

Recebido em: Abr. 10, 2019. Aprovado em: Set. 17, 2019 
other services when the units are closed and lack of priority to care of lived. Conclusion: It is possible to conclude that, through the perception of health professionals, many factors of access are related to the process of work and there is a limited understanding of the elderly must be specific due to physiological changes and social in this population.

Keywords: aged; aging; health services accessibility; Unified Health System; elderly nutrition.

\section{INTRODUÇÃO}

O envelhecimento populacional provoca alterações na vida dos indivíduos e nas estruturas familiares, refletindo na demanda por políticas públicas e na distribuição de recursos na sociedade 1 . As ações de saúde pública relacionadas à pessoa idosa constituem necessidades urgentes, devendo os custos serem considerados como investimentos, pois uma vez que se financiam ações de promoção de envelhecimento saudável mantêm-se melhores níveis de saúde, autonomia, independência e qualidade de vida da população e, consequentemente, a redução de gastos com tratamentos, internações, cuidados e doenças ${ }^{2}$. São considerados idosos longevos ou mais idosos os que têm 80 anos ou mais, sendo esse segmento populacional o que mais cresce no Brasil ${ }^{3}$.

Os sistemas de saúde devem contribuir para que a população envelheça com o melhor estado de saúde possível, demandado por um cuidado estruturado de forma diferente e especial, devido a situações específicas da idade, como doenças crônicas, fragilidades orgânicas e sociais ${ }^{4}$, buscando também a efetivação dos direitos de saúde e cidadania. Nesse sentido, destaca-se a Atenção Primária à Saúde (APS) como provedora central do cuidado 5 . No Brasil, a Estratégia Saúde da Família (ESF) é a estratégia prioritária da APS e deve ter sua atenção voltada para uma perspectiva ampliada de saúde ${ }^{6}$.

Para que a APS possa responder às necessidades de seus usuários, deve ser orientada por atributos essenciais e derivados os quais organizam seu fazer: acesso de primeiro contato, longitudinalidade, integralidade, coordenação do cuidado, atenção à saúde centrada na família, orientação comunitária e competência cultural. $\mathrm{O}$ acesso de primeiro contato é entendido como a acessibilidade e o uso do serviço a cada novo problema ou episódio pelo qual as pessoas buscam atenção à saúde e, em sua ausência, os demais não são efetuados. Envolve a acessibilidade do ponto de vista estrutural, assim como de utilização 7,8 .

No Brasil, houve uma considerável ampliação do acesso na APS, retratada por diferentes indicadores que revelam reduções nas iniquidades em saúde ${ }^{9}$. Sabendo da relação entre desigualdade social e acesso, torna-se necessária a ampliação da cobertura de serviços ${ }^{10}$, o que tem ocorrido no que concerne aos serviços de menor complexidade?

A APS constitui importante porta de entrada do Sistema Único de Saúde (SUS), sendo o contato inicial da população com o serviço de saúde, responsabilizando-se e respondendo pela maioria dos problemas ou necessidades ${ }^{9}$. A ampliação da APS vem provocando impactos significativos na saúde da população, no Brasil e em diversos países, pois tem melhorado os resultados sanitários nas condições crônicas, diminuído as referências a especialistas e aos hospitais, aumentado a eficiência dos sistemas de atenção à saúde, produzido serviços mais custo/efetivos e promovido a satisfação dos usuários ${ }^{11}$.

Dessa forma, é oportuno refletir sobre os possíveis fatores que favorecem ou dificultam o acesso da população idosa à APS, como: número de usuários por equipe, organização da demanda espontânea, localização do estabelecimento, horários e dias de atendimento, infraestrutura para o atendimento, acessibilidade, acolhimento, interações profissional-usuário, modelo de atenção, capacitação e espaços democráticos e reflexivos para reorganizar o processo de trabalho $0^{5,8,12,13}$.

Nesse contexto, o trabalho objetiva analisar a percepção dos profissionais que atuam em ESF, em um município do Sul do Brasil sobre o acesso de longevos à APS.

\section{MÉTODO}

Trata-se de um estudo qualitativo e exploratório com dez profissionais vinculados à ESF. As entrevistas, que ocorreram entre dezembro de 2016 e maio de 2017, foram gravadas, transcritas e analisadas segundo os preceitos da análise temática de conteúdo ${ }^{14}$. A técnica 
explora as unidades temáticas do discurso, em que a presença e a frequência dessas unidades apontam a importância e os modelos de comportamento existentes nas falas.

O estudo foi realizado nas ESFs urbanas do município de Palmeira das Missões, Brasil, situado na região noroeste do Rio Grande do Sul, com 34.328 habitantes ${ }^{4}$. Destes, 4.507 (13,13\%) eram idosos, 581 longevos (1,69\% do total da população e 12,9\% do total de idosos) apresentando um índice de envelhecimento de $65,32^{15}$. A rede de ESF era composta por seis unidades, sete equipes e por 20 profissionais.

Como critérios de inclusão participaram enfermeiros, odontólogos e médicos que atuavam nas ESFs e que aceitaram participar. Não foram incluídos os que estavam em férias e/ou atestado de saúde e/ou afastamento do trabalho durante o período de coletas.

Foi realizada entrevista semiestruturada, constando variáveis sociodemográficas como idade, sexo, cor da pele, escolaridade, estado civil, profissão, tempo de graduação, tempo de trabalho na unidade de saúde, título e área de especialização. Variáveis laborais também foram avaliadas, descritas como: se o profissional era coordenador da equipe, participação no Programa Mais Médicos e grau de satisfação em relação à abordagem aos longevos desenvolvida pela unidade de saúde.

Criou-se um instrumento qualitativo, com perguntas sobre o acesso dos longevos ao serviço de Saúde da Família. As questões abordadas foram: O serviço de saúde fica aberto em todos os momentos em que os longevos necessitam? Quando algum longevo necessita, consegue atendimento rapidamente na sua unidade de saúde? Na sua opinião, como é o acesso dos longevos à unidade de saúde?

Essas questões basearam-se no Primary Care Assessment Tool - versão brasileira para profissionais, o instrumento mais aproximado para a criação de propostas que possam contribuir para a qualificação da Saúde da Família ${ }^{16}$, mas que não compreende as especificidades dos longevos e não é validado para a população idosa. Primeiramente, realizou-se um piloto, para verificar a qualidade do instrumento de coleta. As questões foram aplicadas, gravadas e avaliadas se estavam de acordo com os objetivos da pesquisa. Após constatado que o instrumento estava em conformidade, foi realizada a coleta de dados.

Para os dados sociodemográficos e condições de saúde foi utilizada a análise descritiva, com o Microsoft Excel. As entrevistas foram submetidas a análise temática de conteúdo que, segundo Bardin ${ }^{14}$, é composta pelas seguintes etapas:

1) Pré-análise, em que se realizaram a leitura flutuante dos discursos e a sistematização das ideias iniciais. Cada profissional foi identificado pela letra $\mathrm{P}$, seguido por um número sequencial (P1 ao P10), não necessariamente numerados pela ordem de entrevista ou análise;

2) Exploração do material, com o recorte do texto em unidades temáticas, a codificação e a enumeração;

3) Tratamento dos resultados obtidos e interpretação. Os resultados brutos foram tratados a fim de serem significativos e válidos, realizando-se a síntese, a seleção, as inferências e interpretações dos resultados de acordo com os objetivos do estudo.

Os resultados foram apresentados em nove categorias, de acordo com as falas dos profissionais e respondendo às questões da entrevista: o serviço reserva um turno por semana para a reunião de equipe; o serviço não abre à noite aos finais de semana; priorização; triagem prévia; demanda espontânea; percepção positiva; acessibilidade prejudicada; visita domiciliar; barreira: recepção.

O estudo foi aprovado pelo Comitê de Ética em Pesquisa da Universidade Federal de Santa Maria, parecer $n^{\circ} 1.868 .174$.

\section{RESULTADOS}

A idade dos participantes variou de 27 a 47 anos, com média de $33,7 \pm 7,16$ e maior frequência abaixo de 40 anos (80\%), do sexo feminino (80\%), todos autorreferiram ser de

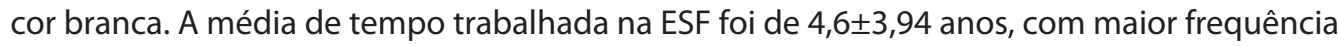


entre um a cinco anos (60\%). Dos entrevistados, $40 \%$ eram coordenadores de equipes, enfermeiros. Quanto à satisfação com o trabalho, $90 \%$ referiram estar parcialmente satisfeitos e $10 \%$ totalmente satisfeito. Das sete equipes estudadas, três possuíam profissionais de saúde vinculados ao Programa Mais Médicos.

Em relação à formação acadêmica, houve maior frequência de enfermeiros (40\%), com 5 a 10 anos de atuação (60\%), com média de 10 anos e 4 meses $\pm 7,05$. Possuíam especialização $80 \%$, dos quais $20 \%$ em ESF, $20 \%$ em educação em saúde e gestão pública; não foi identificada formação em envelhecimento humano.

O acesso representa a entrada no sistema de saúde, condição indispensável em relação aos demais atributos. O Quadro 1 apresenta os resultados de acordo com as perguntas realizadas, as categorias e as principais falas que elucidam as temáticas.

As unidades não estão abertas para atendimento em todos os momentos que os longevos necessitam. Como opção alternativa, há outros espaços que realizam atendimento, tais como o Postão, hospital, Serviço de Atendimento Móvel de Urgência (SAMU). Apesar de ter um serviço

Quadro 1. Categorias e falas que elucidaram as questões sobre o acesso. Profissionais da Estratégia de Saúde da Família. Rio Grande do Sul - RS, Brasil, 2016/2017.

\begin{tabular}{|c|c|c|}
\hline Pergunta & Categoria & Trechos das falas \\
\hline \multirow{2}{*}{$\begin{array}{l}\text { O serviço de saúde } \\
\text { fica aberto em } \\
\text { todos os momentos } \\
\text { em que os longevos } \\
\text { necessitam? }\end{array}$} & $\begin{array}{l}\text { O serviço reserva } \\
\text { um turno por } \\
\text { semana para a } \\
\text { reunião de equipe. }\end{array}$ & $\begin{array}{l}\text { "Apenas em um turno de atendimento [...] é separado para } \\
\text { o processo interno, reunião de equipe, organização do } \\
\text { processo de trabalho." (P5). }\end{array}$ \\
\hline & $\begin{array}{l}\text { O serviço não abre } \\
\text { à noite aos finais de } \\
\text { semana. }\end{array}$ & $\begin{array}{l}\text { [...] Tem o Postão, mas ele não fica próximo da nossa } \\
\text { área, é bem distante. É um fator dificultador [...]. E tem o } \\
\text { hospital, que também é distante do posto [...]. (P5). }\end{array}$ \\
\hline \multirow{2}{*}{$\begin{array}{l}\text { Quando algum } \\
\text { longevo necessita, } \\
\text { consegue } \\
\text { atendimento } \\
\text { rapidamente na sua } \\
\text { unidade de saúde? }\end{array}$} & Priorização & $\begin{array}{l}\text { "A gente procura priorizar [...] independente de ele ter } \\
\text { horário para às } 9, \text { mas chegou às } 8 \text {, a gente procura } \\
\text { atender [...], atende antes." (P5). } \\
\text { "Por uma questão de humanidade, a gente sempre prioriza } \\
\text { a pessoa idosa }[. . .] \text { A gente prioriza mesmo que outra } \\
\text { pessoa tenha chegado mais cedo..." (P6). }\end{array}$ \\
\hline & Triagem prévia & $\begin{array}{l}\text { "A gente sempre faz uma avaliação, uma triagem. Logo que } \\
\text { necessário, ele é atendido [...] A partir daí a gente vê se tem } \\
\text { prioridade ou não." (P1). } \\
\text { "O longevo tem acesso prioritário de acordo com o caso, se } \\
\text { a gente vê que é muito velhinho ou não consegue esperar } \\
\text { muito tempo [...]." (P3). }\end{array}$ \\
\hline \multirow{5}{*}{$\begin{array}{l}\text { Na sua opinião, } \\
\text { como é o acesso } \\
\text { dos longevos à } \\
\text { unidade de saúde? }\end{array}$} & $\begin{array}{l}\text { Demanda } \\
\text { espontânea }\end{array}$ & $\begin{array}{l}\text { "A unidade trabalha com livre demanda para urgência e } \\
\text { emergência [...] seis fichas que são entregues aos pacientes } \\
\text { que chegam pedindo [...]." (P9). }\end{array}$ \\
\hline & Percepção positiva & $\begin{array}{l}\text { "O acesso é facilitado. Dá briga, mas a gente quer tentar } \\
\text { implantar isso aqui." (P6). }\end{array}$ \\
\hline & $\begin{array}{l}\text { Acessibilidade } \\
\text { prejudicada }\end{array}$ & $\begin{array}{l}\text { "Acho que é dificil a entrada e o acesso que está feio, } \\
\text { na frente da unidade é ruim. Aquela calçada está toda } \\
\text { quebrada e aí dificulta o acesso, mas aqui dentro acho que } \\
\text { não é tanto assim, porque é tudo plano né, não tem escada, } \\
\text { os corredores são mais larguinhos [...]." (P10). }\end{array}$ \\
\hline & Visita domiciliar & $\begin{array}{l}\text { "VD a gente faz bastante, geralmente é idoso, que não } \\
\text { consegue vir até aqui, [...] que não consegue se locomover } \\
\text { muito bem." (P3). }\end{array}$ \\
\hline & Barreira: recepção & $\begin{array}{l}\text { "[...] não tem um recepcionista e acho que faz falta ter uma } \\
\text { pessoa fixa que vá acolher, usar protocolo e falar a mesma } \\
\text { língua." (P1). }\end{array}$ \\
\hline
\end{tabular}


ofertado para o terceiro turno, o acesso não é facilitado em decorrência da distância entre a unidade central e a casa dos usuários.

O deslocamento até as unidades também pode estar comprometido para muitos idosos, pela redução da reserva funcional e da realização das atividades de vida diária. Desse modo, ressalta-se a importância das Visitas Domiciliares (VDs) como forma de acesso.

\section{DISCUSSÃO}

A perspectiva dos profissionais de saúde das ESFs em relação ao cuidado do longevo constitui um tema novo, visto que esse é o segmento que mais cresce no Brasil e no mundo e que demanda por políticas públicas e cuidados especiais.

As ESFs participantes do estudo eram todas coordenadas por enfermeiros, fato que pode estar relacionado com a formação desses, que inclui o cuidado e a gestão ${ }^{17}$.

A maioria dos profissionais esteve parcialmente satisfeita com o serviço que ofertava. A satisfação no trabalho na APS é influenciada pelos seguintes fatores: afinidade pelo trabalho, resolubilidade da assistência, trabalho em equipe, vínculo entre a equipe e com os usuários ${ }^{17}$.

A demanda superior à capacidade de atendimento nas unidades está atrelada ao maior número de atribuições e pode influenciar na satisfação da equipe. É comum a criação de novas ações, programas e funções sob a responsabilidade das equipes de saúde, gerando sobrecarga de trabalho ${ }^{18}$. Por isso, espaços de Educação Permanente em Saúde devem ser valorizados para a efetividade das ações da APS.

Como facilitadores do acesso destacou-se a percepção positiva dos profissionais, com realização das VDs, acolhimento e escuta, acesso por demanda espontânea e atendimento rápido aos longevos. Por outro lado, as barreiras constituíram-se pelas unidades não estarem abertas em todos os momentos em que os longevos necessitavam, ausência de recepcionista, distância de outros serviços quando a ESF estava fechada e alguns profissionais que não priorizam o atendimento aos longevos.

Há poucos estudos que exploram o acesso dos longevos aos serviços de saúde e que, em muitos casos, esse acesso é desigual ${ }^{19}$. Maior renda e escolaridade estiveram associadas à utilização e acesso a consultas ${ }^{20}$.

Considerando que um melhor acesso à APS está relacionado à redução de visitas à emergência ${ }^{21-23}$, priorizar os atendimentos e os serviços aos longevos pode diminuir também internações de condições sensíveis à APS ${ }^{21-23}$.

A ESF deve ser a entrada preferencial no sistema de saúde, garantir o acesso e a acessibilidade dos serviços de APS e ter como componentes organizacionais o funcionamento, 0 acolhimento, a organização da agenda, as formas de atendimento e acessibilidade ${ }^{24}$. Em relação à acessibilidade temporal, as USs observadas poderiam ser classificadas como mais acessíveis, pois abrem cinco dias na semana e têm carga horária diária de oito horas ou mais ${ }^{24}$. Contudo, apresentam limitações por encerrarem o atendimento às 17 horas e não estarem abertas nos finais de semana, configurando como importante barreira para o acesso. Verificou-se que há serviços substitutivos no horário em que as ESFs estão fechadas, porém encontram-se mais distantes das residências.

Investir em acesso aos serviços de APS fora de horário ou eliminar fatores que impedem as pessoas de usá-los, como, por exemplo, problemas financeiros ou deslocamento, podem ser benéficos e reduzir as idas aos serviços emergenciais ${ }^{20}$. As barreiras no acesso temporal podem gerar uma baixa resolutividade e até colapso em outros pontos de atenção da rede de saúde ${ }^{25}$.

Nos serviços de saúde, os idosos devem ter a prioridade de atendimento, sendo os longevos ter preferência especial ${ }^{26}$. Percebeu-se que os trabalhadores ainda possuem resistência ou desconhecimento para reconhecer tal direito, posturas que limitam o acesso e o cuidado prestado a um público mais vulnerável.

Por outro lado, o acolhimento, associado à escuta, foi apontado como ferramenta de acesso dos longevos e possibilita a autonomia, a cidadania e a corresponsabilização na produção do cuidado à saúde ${ }^{12,13}$. 
Apesar dos profissionais deste estudo afirmarem que os longevos conseguem atendimento rapidamente, há diminuição das capacidades sensório-perceptivas em decorrência do processo de envelhecimento, afetando a comunicação, indispensável para obtenção de informações para a condução terapêutica. Nesse sentido, o ato de acolher está relacionado ao processo comunicativo, que permite que as pessoas se tornem acessíveis umas às outras por meio do compartilhamento de sentimentos, opiniões, experiências e informações ${ }^{12,13}$.

Algumas vezes, não se percebe a presença de incapacidades funcionais nos idosos, tornando-se imprescindível a avaliação multidimensional enquanto método de avaliação integral, podendo direcionar intervenções propostas, como a construção do Plano de Cuidados $(\mathrm{PC})$, visando a melhoria da saúde, atuando no processo de fragilização, prevenção, controle, reabilitação ou conforto ${ }^{27-29}$.

O conceito de PC pode ser associado ao de Projeto Terapêutico Singular (PTS), que organiza o processo de trabalho e gerencia o cuidado. Constitui-se em um plano de ação que considera todos os fatores envolvidos no processo de adoecimento, formulando uma estratégia de intervenção, pactuada com o usuário ${ }^{30}$.

No PTS há coprodução e cogestão do processo terapêutico de indivíduos ou coletivos em situação de vulnerabilidade, contribuindo para a diversificação das ofertas nos serviços de saúde ${ }^{31,32}$. Compõe um projeto maior, a Linha de Cuidado Integral $(\mathrm{LCl})$, definida como fluxos de ações assistenciais, técnicas e de educação, que pressupõe uma rede articulada, referenciada e com um sistema de informações em sintonia. Deve ser centrada no usuário e permeia a sensibilidade para captar essa relação, associada a concepções sobre saúde, doença e o bem viver, assim como nas possibilidades de articulação entre conhecimento e itinerários terapêuticos ${ }^{31,32}$.

Cabe ressaltar que nenhum profissional mencionou a avaliação multidimensional, o PTS ou a LCl, ou seja, o planejamento e o desenvolvimento das ações não são orientados pela utilização dessas ferramentas de processo de trabalho. É importante a adoção de uma compreensão multidimensional para avaliar e identificar as necessidades e especificidades de cada pessoa idosa, do ponto de vista clínico, psicossocial e funcional a fim de direcionar as intervenções adequadas e o caminho a ser percorrido junto às Redes de Atenção à Saúde (RAS) e às redes intersetoriais, buscando a manutenção ou a recuperação da independência e da autonomia possíveis para melhor qualidade de vida no processo de envelhecimento ${ }^{33}$.

Embora as VDs não se constituam como porta de entrada da APS e sim um dispositivo de cuidado, podem contribuir para o acesso, especialmente aos longevos. Um estudo ${ }^{10}$ aponta que as VDs favoreceram o acesso aos idosos mais pobres. Desse modo, os profissionais utilizam essa potente ferramenta, mas cabe refletir que poderia ter maiores impactos associados com a LCl ou o PTS para longevos, o que levaria a um melhor planejamento para a realização das VDs e outras ações relacionadas ao acesso.

$\mathrm{Na}$ perspectiva de $\mathrm{LCl}$, a porta de entrada deve ser o espaço facilitador para que o idoso se sinta protegido, amparado e acolhido, constituindo o primeiro contato, em que o usuário é informado de todas as possibilidades e dos percursos assistenciais ${ }^{31}$. Nessa lógica, constata-se a importância de recepcionista, inexistente nos serviços avaliados e que, para lidar com essa situação, alguns profissionais revezavam-se para atender a população, prejudicando o acolhimento, gerando sobrecarga de trabalho e, consequentemente, representando uma barreira para o acesso.

O estudo apresenta limitações, como estar restrito a um município e a um tipo de serviço, assim como o número reduzido de participantes, justificado pelo estudo ser qualitativo. Entretanto, tem o potencial de aprofundar e colaborar com o olhar dos profissionais de ESFs em relação ao acesso e seus dispositivos concernentes aos longevos na APS, um tema pouco abordado, assim como apresenta implicações práticas ao ampliar a visão acerca dos mecanismos de acesso ofertados à população longeva, podendo melhorar os serviços de saúde prestados a essa população, que apresenta maior vulnerabilidade, além de propiciar condições de trabalho mais satisfatórias aos profissionais da APS.

O estudo pode colaborar para a estruturação de um acesso facilitado para longevos e, consequentemente, para a população em geral, a fim de incorporar a acessibilidade e a 
usabilidade dos serviços de saúde da APS. Os resultados desta pesquisa podem ser utilizados para a construção e planejamento de atividades de educação permanente em saúde, com os profissionais da APS, relacionada ao envelhecimento humano; para o fomento da discussão no âmbito do controle e mobilização social em busca de condições de envelhecimento mais dignas e justas para a população brasileira; na esfera da gestão, na construção de ações, serviços e redes que proporcionem à pessoa idosa itinerários terapêuticos pautados no cuidado integral, na humanização e na APS como ordenadora desse cuidado; e, por fim, para uma formação acadêmica comprometida com a realidade social e que vislumbre o envelhecimento como um direito de todo cidadão brasileiro a partir do fortalecimento do SUS.

Considerando que os longevos constituem uma população em maior vulnerabilidade, com necessidades diferenciadas e que demandam maiores cuidados, torna-se fundamental o uso de ferramentas como a avaliação multidimensional, acolhimento, PCl e de PTS para ampliar o acesso e tecer uma rede de cuidado pautada na humanização, que vise uma APS fortalecida e ordenadora da atenção.

\section{REFERÊNCIAS}

1. Peixoto N, Lima LCV, Bittar CML. Percepções sobre qualidade de vida entre idosos que participam de uma Universidade Aberta para Maturidade. Acta Scientiarum Human and Social Sciences. 2017;39(2):209-16. http://dx.doi.org/10.4025/actascihumansoc.v39i2.33089.

2. Organizaçao Mundial de Saúde. Resumo do Relatório Mundial de Envelhecimento e Saúde. Genebra: OMS; 2015.

3. Navarro JH, Andrade FP, Paiva TS, Silva DO, Gessinger CF, Bós ÂJ. Percepção dos idosos jovens e longevos gaúchos quanto aos espaços públicos em que vivem. Cien Saude Colet. 2015;20(2):461-70. http://dx.doi. org/10.1590/1413-81232015202.03712014. PMid:25715140.

4. Veras, RP, Oliveira, M. Envelhecer no Brasil: a construção de um modelo de cuidado. Cienc Saude Col. 2018; 23(6):1929-36. http://dx.doi.org/10.1590/1413-81232018236.04722018.

5. Macinko J, Harris MJ. Brazil's family health strategy--delivering community-based primary care in a universal health system. N Engl J Med. 2015;372(23):2177-81. http://dx.doi.org/10.1056/NEJMp1501140. PMid:26039598.

6. Peixoto MT, Carvalho RC, Vilasboas ALQ. Projeto Terapêutico Familiar: uma tecnologia de gestão do cuidado na saúde da família. Rev Saúde Col. 2017;7(2):35-43. http://dx.doi.org/10.13102/rscdauefs.v7i2.1498.

7. Starfield B. Atenção Primária: equilíbrio entre necessidades de saúde, serviços e tecnologia. Brasília: UNESCO, Ministério da Saúde; 2002.

8. Damaceno AN, Bandeira D, Hodali N, Weiller TH. Acesso de Primeiro Contato na Atenção Primária à Saúde: revisão integrativa. Rev. APS. 2016;19(1):122-38.

9. Malta DC, Santos MA, Stopa SR, Vieira JE, Melo EA, Reis AA. A Cobertura da Estratégia de Saúde da Família (ESF) no Brasil, segundo a Pesquisa Nacional de Saúde, 2013. Cien Saude Colet. 2016 fev;21(2):327-38. http://dx.doi.org/10.1590/1413-81232015212.23602015. PMid:26910142.

10. Almeida APSC, Nunes BP, Duro SMS, Facchini LA. Determinantes socioeconômicos do acesso a serviços de saúde em idosos: revisão sistemática. Rev Saude Publica. 2017 maio;51:50. http://dx.doi.org/10.1590/ s1518-8787.2017051006661. PMid:28513761.

11. Mendes EV. Entrevista: a abordagem das condições crônicas pelo Sistema Único de Saúde. Cien Saude Colet. 2018;23(2):431-6. http://dx.doi.org/10.1590/1413-81232018232.16152017. PMid:29412401.

12. Coutinho LRP, Barbieri AR, Santos MLM. Acolhimento na Atenção Primária à Saúde: revisão integrativa. Saúde Debate. 2015;39(105):2071-85. http://dx.doi.org/10.1590/0103-110420151050002018.

13. Ferraz LR, Moreno CA, Rodrigues TS, Lopes AOS. Acolhimento: uma prática real ou mera ideologia? Rev Bras Pesq Saúde. 2015;17(2):122-30.

14. Bardin L. Análise de conteúdo. São Paulo: Edições 70; 2011.

15. Brasil. Ministério da Saúde. SISAP Idoso - Sistema de Indicadores de Saúde e Acompanhamento de Políticas do Idoso. Painel de Indicadores Municipais. Brasília: Ministério da Saúde; 2011. Disponível em: https://sisapidoso.icict.fiocruz.br/painel-de-indicadores-municipais.

16. Fracolli LA, Gomes MFP, Nabão FRZ, Santos MS, Cappellini VK, Almeida ACC. Instrumentos de avaliação da Atenção Primária à Saúde: revisão de literatura e metassíntese. Cien Saude Colet. 2014 dez;19(12):485160. http://dx.doi.org/10.1590/1413-812320141912.00572014. PMid:25388193. 
17. Lima L, Pires DEP, Forte ECN, Medeiros F. Satisfação e insatisfação no trabalho de profissionais de saúde da atenção básica. Escola Anna Nery Revista de Enfermagem. 2017;18(1):17-24. http://dx.doi. org/10.5935/1414-8145.20140003.

18. Tesser CD, Norman AH. Repensando o acesso ao cuidado na Estratégia Saúde da Família. Saude Soc. 2014;23(3):869-83. http://dx.doi.org/10.1590/S0104-12902014000300011.

19. Salway SM, Paune N, Rimmer M, Buckner S, Jordan H, Adams J, Waters K, et al. Identifying inequitable healthcare in older people: systematic review of current research practice. Int J Equity Health. 2017;16(1). http://dx.doi.org/10.1186/s12939-017-0605-z. PMid: 28697768.

20. Zanesco C, Bordin D, Santos CB, Müller EV, Fadel CB. Fatores que determinam a percepção negativa da saúde de idosos brasileiros. Rev Bras Geriatr Gerontol. 2018;21(3):293-303. http://dx.doi.org/10.1590/198122562018021.170210.

21. van den Berg MJ, van Loenen T, Westert GP. Accessible and continuous primary care may help reduce rates of emergency department use. An international survey in 34 countries. Fam Pract. 2016;33(1):42-50. http://dx.doi.org/10.1093/fampra/cmv082. PMid:26511726.

22. Bós AJG, Kimura AY. Internações por Condições Sensíveis à Atenção Primária em idosos com 80 anos ou mais em um Hospital Universitário do Rio Grande do Sul. Revista da AMRIGS. 2015;59(2):106-11. http:// dx.doi.org/10.1590/S0034-8910.2014048005133.

23. Marques AP, Montilla DE, Almeida WS, Andrade CL. Internação de idosos por condições sensíveis à atenção primária à saúde. Rev Saude Publica. 2014 out;48(5):817-26. http://dx.doi.org/10.1590/S00348910.2014048005133. PMid:25372173.

24. Fausto $M C R$, Giovanella L, Mendonça MHM, Seidl H, Gagno J. A posição da Estratégia Saúde da Família na rede de atenção à saúde na perspectiva das equipes e usuários participantes do PMAQ-AB. Saúde debate. 2014;38(N. esp.):13-33. http://dx.doi.org/10.5935/0103-1104.2014S003.

25. Araújo LUA, Gama ZAS, Nascimento FLA, Oliveira HFV, Azevedo WM, Almeida JHJB. Avaliação da qualidade da atenção primária à saúde sob a perspectiva do idoso. Cien Saude Colet. 2014;19(8):3521-32. http:// dx.doi.org/10.1590/1413-81232014198.21862013.

26. Lima CCS. Lei no 13.466/2017 e a alteração do estatuto do idoso: uma contradição ao rol de prioridade especial para pessoas com mais de 80 anos. Cad Ibero-Amer Dir Sanit. 2018;7(1):147-62. http://dx.doi. org/10.17566/ciads.v7i1.462.

27. Nunes JD, Saes MO, Nunes BP, Siqueira FCV, Soares DC, Fassa MEG, et al. Indicadores de incapacidade funcional e fatores associados em idosos: estudo de base populacional em Bagé, Rio Grande do Sul. Epidemiol Serv Saude. 2017;26(2):295-304. http://dx.doi.org/10.5123/S1679-49742017000200007. PMid:28492771.

28. Rodrigues RMC, Silva CFR, Loureiro LMJ, Silva SMDT, Crespo SSS, Azeredo ZAS. Os muito idosos: avaliação funcional multidimensional. Rev Enf Ref. 2015 jun;(5):65-74. http://dx.doi.org/10.12707/RIV14040.

29. Veras, R. Linha de cuidado para o idoso: detalhando o modelo. Rev Bras Geriatr Gerontol. 2016;19(6):887905. https://doi.org/10.1590/1981-22562016019.160205.

30. Oliveira MR, Veras RP, Cordeiro HA, Pasinato MT. A mudança de modelo assistencial decuidado ao idoso na Saúde Suplementar: identificação de seus pontos-chave e obstáculos para implementação. Physis. 2016;26(4):1383-94. http://dx.doi.org/10.1590/s0103-73312016000400016.

31. Silva NE, Sancho LG, Figueiredo WS. Entre fluxos e projetos terapêuticos: revisitando as noções de linha do cuidado em saúde e itinerários terapêuticos. Cien Saude Colet. 2016 mar;21(3):843-52. http://dx.doi. org/10.1590/1413-81232015213.08572015. PMid:26960096.

32. Santos CTB, Barros IS, Amorim ACCLÁ, Rocha DG, Mendonça AVM, Sousa MF. A integralidade no Brasil e na Venezuela: similaridades e complementaridades. Cien Saude Colet. 2018;23(4):1233-40. http://dx.doi. org/10.1590/1413-81232018234.16122016.

33. Brasil. Ministério da Saúde. Secretaria de Atenção à Saúde. Departamento de Ações Programáticas e Estratégicas. Orientações técnicas para a implementação de Linha de Cuidado para Atenção Integral à Saúde da Pessoa Idosa no Sistema Único de Saúde - SUS. Brasília: Ministério da Saúde; 2018. 